\title{
Detection of Adulteration and Quality Evaluation of Market Milk and Raw Milk Collected from Market in Varanasi City
}

\author{
Dwarki Lal $^{1 *}$, D. C. Rai ${ }^{1}$, Vinod Bhateshwar ${ }^{1}$, Hitesh Muwal $^{2}$ and Govind ${ }^{3}$ \\ ${ }^{1}$ Department of Animal Husbandry and Dairying, \\ Banaras Hindu University, Varanasi (U.P.) India \\ ${ }^{2}$ Department of Animal Production, Maharana Pratap University of \\ Agriculture and Technology, Udaipur (Raj.) India \\ ${ }^{3}$ Department of Animal Husbandry and Dairying, Chandra Shekhar Azad \\ University of Agriculture and Technology, Kanpur (U.P.) India \\ *Corresponding author
}

K e y w o r d s
Adulteration,
Market milk, Raw
milk, Branded,
Organized dairy
farms and
Traditional vendors
Article Info
Accepted:
25 February 2020
Available Online:
10 March 2020

\section{Keywords}

Adulteration, Market milk, Raw milk, Branded Organized dairy

endors

25 February 2020

Available Online:

\section{A B S T R A C T}

The present investigation was conducted to assess the presence of adulterants and contaminants in market milk and raw milk around Varanasi city from branded, organized dairy farms and traditional vendors. The trial work has been approved out during the period of 1 February 2019 to 20 May 2019. Four milk samples were collected from each branded, organized dairy farm and traditional vendors milk centers 250- and 500-ml. quantity was collected and decontaminated throwaway plastic bottles with screw cap and each bottle was coded. The bottles were filled in ice box and proximately ecstatic to further testing. Samples were collected randomly encompassing Varanasi city. Out of $168^{\text {th }}$ milk samples, $44.04 \%$ milk samples were found with water, $4.16 \%$ with urea, $22.02 \%$ with starch, $36.30 \%$ with sugar, $11.30 \%$ with glucose, $26.78 \%$ with salt and $7.38 \%$ with neutralizers. This study was conducted to know the extent of adulteration found in traditional vendors milk than organized dairy farm and branded milk samples. In this research, no adulteration was found in branded milk samples. But adulteration was found in traditional vendors and organized dairy farm milk samples. Branded milk was found in good quality for human health. The traditional vendors and organize dairy farm lack of suitable technical and educational information causes inferior quality of milk and indigenous milk especially in unorganized sector these are the main reasons for deterioration of milk. Also, the demand for the value-added milk is continuously increasing because of consumer awareness about health and nutrition. So, it is important to ensure the consumer about the quality, health and nutrition claims of such milk. This can be achieved by rapid analytical methods and techniques Research. 


\section{Introduction}

Milk is a defined as the whole, fresh, clean, lacteal secretion obtained from complete milking of one or more healthy animals excluding that obtained within fifteen days before or five days after calving or such periods as may be necessary to render the milk practically colostrum free and containing the minimum prescribed percentage of the milk fat and solids not fat so as to confirm the legal standards or other requirements (FSSAI, 2011). Milk is a completed food that invariable improves the country's food and nutritive security. Milk is a source of essential nutrients such fats, proteins, as carbohydrates, minerals and vitamins.

Being a major constituent of the diet, quality Assurance of milk is considered essential to the health and welfare of a community. However, the area of interest of developing nations is to provide enough food to the people rather than quality and hygiene of the food (Ellis and Sumberg et al., 1998). Milk is a balanced food stuff with a very low microbial load at the time milking but various contaminants such as pathogenic organisms, antibiotics, pesticides, mycotoxins etc. enter during various stages of production and processing.

India ranks first in the world, in milk production producing 176.35 million tonnes (2017-18) accounting $21.32 \%$ of the total world milk production (NDDB 2017-18). India milk production and distribution system not improved and only $10 \%$ of the milk is handled by organising sector. Nearly $46 \%$ of milk produced in India is consumed as liquid milk and so there is every chance for adulteration. As there is rapid growth of population, scattered colonisation and urbanisation, milk consumption is increased but not the milk supply (Awan et. al. 2014).
In the recent times, media has highlighted many instances of adulteration of milk and milk products with various kinds of adulterants. In general, every milk industry in India is facing problem of adulterated milk at reception dock. Therefore, milk collection centres need simple tests for detection of adulteration. The practice of adulteration of milk is as old as history, and is one of the major problems that stand against the progress of dairying in India and may also have detrimental effect on our export of dairy products. The image of milk has been considerably deteriorated due to its adulteration with harmful chemicals. With the analytical methods developed for most of the adulterants, unscrupulous traders are finding more innovative ways to adulterate the milk with cheaper ingredients.

A survey by FSSAI in 2012, 68\% milk samples was found to be adulterated in which $31 \%$ were from rural areas. Of these $16.7 \%$ were packet or branded milk and rest were loose milk samples from dairies. In the urban areas, $68.9 \%$ milk was found to be adulterated with water, detergent, urea and skim milk powder. Water is the most commonly used adulterant to increase the volume of milk.

\section{Materials and Methods}

\section{Place of work}

This study was directed in the Department of Animal Husbandry and Dairying, Institute of Agricultural Sciences, Banaras Hindu University, Varanasi, Uttar Pradesh. The trial work has been approved out during the period of 1 February 2019 to 20 May 2019.

\section{Collection of milk samples}

The market milk and raw milk samples were collected from different branded, organized 
dairy farm and vendors of Varanasi city. Four milk samples were collected from each branded, organized dairy farms, traditional milk vendors milk centres 250 and $500 \mathrm{ml}$ quantity was collected from local market Varanasi city of Uttar Pradesh state, in fresh and decontaminated throwaway plastic bottles with screw cap and each bottle was coded. The bottles were filled in ice box and proximately ecstatic to further testing. All milk samples will be occupied from above $168^{\text {th }}$ branded, organized dairy farm and traditional milk vendors samples four replication for judging the adulteration and quality properties of milk.

\section{Preparation of chemicals and media}

All the chemicals used in the present study, Department of Animal Husbandry and Dairying lab, were ready consulting to standard procedures. A standard milk adulteration chemical media was used for detection of adulterants, neutralizers, preservatives and thickening agents, alizarine, formalin, urea, starch, neutralizers, detergents, sodium chloride, skimmed milk powder, sugar (sucrose), glucose (dextrose) and hydrogen peroxide maltose, ammonium sulphate, proteins, pond water and boric acid.

\section{Methods of adulteration analysis in market milk and raw milk}

\section{Synthetic milk constituents}

Detection of added urea in milk by qualitative method

This process is based on the principle that urea forms a yellow complex with DMAB in a low acidic solution at room temperature.

\section{Reagent}

DMAB reagent $(1.6 \%, \mathrm{w} / \mathrm{v})$ : dissolve $1.6 \mathrm{~g}$
DMAB in $100 \mathrm{ml}$ ethyl alcohol and add $10 \mathrm{ml}$ concentrate HCL.

\section{Procedure}

Mix $1 \mathrm{ml}$ of milk with $1 \mathrm{ml}$ of $1.6 \%$ DMAB reagent. Dissimilar yellow colour is observed in milk containing added urea. The control (normal milk) displays a slight yellow colour due to presence of natural urea. The limit of detection of method is $0.2 \%$.

Detection of ammonium compounds in milk

\section{Reagents}

Added the $2 \%$ sodium hydroxide, $2 \%$ sodium hypochlorite and 5\% phenol solution.

\section{Procedure}

Take $1.0 \mathrm{ml}$ of milk add $0.5 \mathrm{ml}$ of $2 \%$ sodium hydroxide, $0.5 \mathrm{ml}$ of $2 \%$ sodium hypochlorite and $0.5 \mathrm{ml}$ of $5 \%$ phenol solution. Heat for 20 seconds in boiling water bath, bluish colour turns deep blue in incidence of ammonium sulphate. The expansion of pink colour shows that the sample is free from ammonium sulphate.

\section{Detection of nitrates (pond water) in milk}

\section{Reagent}

Diphenylamine $(2 \%, \mathrm{w} / \mathrm{v}$, in sulfuric acid): weigh $2 \mathrm{~g}$ of diphenylamine and dissolve It in sulfuric acid to obtain final volume of $100 \mathrm{ml}$.

\section{Procedure}

Take $2 \mathrm{ml}$ of milk in a test tube. Solution the tube with the milk and drain the milk after the test tube. Add two-three drops of the substance along the side of the test tube. Note the developed colour. Deep blue colour determination be formed in incidence of 
nitrate in the milk sample. Pure milk sample determination not develop any colour.

Detection of neutralizers in milk by Rosalie acid method

\section{Reagents}

Rosalic acid solution $(0.05 \%$, w/v): first prepare $60 \%(\mathrm{v} / \mathrm{v})$ ethyl alcohol solution by mixing $60 \mathrm{ml}$ ethyl alcohol (95\%) and $40 \mathrm{ml}$ distilled water. Weigh $50 \mathrm{mg}$ of rosalic acid powder and dissolve it in small quantity of $60 \%$ ethyl alcohol and make up the volume to $100 \mathrm{ml}$ with $60 \%$ ethyl alcohol.

\section{Procedure}

Take $2 \mathrm{ml}$ milk sample in a test tube and add $2 \mathrm{ml}$ rosalic acid solution. Mix the contents. Unknown alkali is present in milk, a rose red colour seems while clean milk shows only a brownish colour.

\section{Thickening agents}

Detection of starch in milk by qualitative method

\section{Reagent}

Iodine solution: dissolve $2.6 \mathrm{~g}$ of iodine and 3 $\mathrm{g}$ of potassium iodide in a sufficient quantity of water and make up to $200 \mathrm{ml}$.

\section{Procedure}

Take about $5 \mathrm{ml}$ of milk in a test tube. Take to boiling condition and permit the test tube to cool to room temperature. Add the 1-2 drops of iodine solution to the test tube. Development of blue colour designates incidence of starch which disappears after sample is boiled and reappears on cooling. The limit of recognition of method is $0.02 \%$.
Detection of cane sugar in milk by qualitative method: modified Seliwanoff's method

\section{Reagent}

Resorcinol solution $(0.5 \%)$ : weigh $0.5 \mathrm{~g}$ of resorcinol in about $40 \mathrm{ml}$ of distilled water. Add $35 \mathrm{ml}$ of concentrated HCL (12 n) to it and make up the volume to $100 \mathrm{ml}$ using distilled water.

\section{Procedure}

Take $1 \mathrm{ml}$ of milk in a test tube. Add $1 \mathrm{ml}$ of resorcinol solution and mix. Place the tube in boiling water bath for $5 \mathrm{~min}$. Remove the tube and detect the colour. Presence of deep red colour indicates occurrence of sucrose, or a ketose sugar. In pure milk samples no such red colour is developed and sample remains white in nature. The limit of detection of method is $0.1 \%$.

\section{Detection of glucose in milk by qualitative method}

\section{Reagents}

Modified Barford's reagent: dissolve $24 \mathrm{~g}$ of copper acetate in $450 \mathrm{ml}$ of boiling distilled water. Add $25 \mathrm{ml}$ of $8.5 \%$ acetic acid, shake, cool to room temperature and bottle. Make up to $500 \mathrm{ml}$. After sedimentation filter the reagent and store in dark coloured. Phosphomolybdic acid: take $35 \mathrm{~g}$ ammonium molybdate and $5 \mathrm{~g}$ sodium tungstate in a large beaker; add $200 \mathrm{ml}$ of $10 \% \mathrm{NAOH}$ solution and $200 \mathrm{ml}$ water.

Boil strongly (20-60 min) thus as to eliminate closely entire of ammonia. Chickened elimination of ammonia with the help of red litmus paper. Cool, thinned with water to near $350 \mathrm{ml}$. Add $125 \mathrm{ml}$ concentrated $\mathrm{H}_{2} \mathrm{PO}_{4}$ (85\%) and dilute additional to $500 \mathrm{ml}$. 


\section{Procedure}

Take $1 \mathrm{ml}$ of milk sample in a test tube. Add $1 \mathrm{ml}$ of modified Barford's reagent. Heat the mixture for exact $3 \mathrm{~min}$ in a boiling water bath. Quickly cool under tap water. Add one $\mathrm{ml}$ of phosphomolybdic acid reagent to the disorganized solution. Detect the colour. Instant development of deep blue colour after addition phosphomolybdic acid reagent indicates the presence of added glucose in the milk sample. In case of pure milk, only faint bluish colour can be observed due to the dilution of Barford's reagent. The limit of detection of method is $0.1 \%$.

\section{Statistical analysis}

Data related to adulteration and quality evaluation of market milk and raw milk were statistically analyzed using the one-way analysis of variance (SPSS version 21.0) for completely randomized design. All statement of significant differences was based on the 0.05 probability level. Significant differences among treatment, within the experiment, were analyzed using the SPSS statistical software program.

\section{Results and Discussion}

\section{Adulteration in market \& raw milk samples}

A total 168 milk samples were tested for adulteration by chemical reagents. Out of 168 milk samples 56 milk samples were collected from branded, 56 milk samples from organized dairy farm and traditional vendor 56 milk samples were collected from Varanasi city.

\section{Adulteration in branded full cream milk samples}

All 28 milk samples from branded were found to be negative for urea, ammonium fertilizer, nitrate fertilizers, starch, salt, neutralizer and hydrogen peroxide.

\section{Adulteration in branded toned milk samples}

All 28 milk samples from branded were found to be negative for urea, ammonium fertilizer, nitrate fertilizers, starch, salt, neutralizer and hydrogen peroxide.

\section{Adulteration in organized dairy farm cow milk samples}

All 28 samples from organized dairy farm were found to be negative for urea, ammonium fertilizer, nitrate fertilizers, starch $\&$ neutralizer and hydrogen peroxide, while 4 milk samples were positive for urea, 7 milk samples positive for starch and 13 samples were positive for water, 9 milk samples were positive for sugar, 3 milk samples were positive for glucose, 5 samples was found positive for salt and 6 milk samples were positive for neutralizers.

Out of total analysis organized dairy farm cow milk samples, $14.28 \%$ samples were detected positive for urea, $25.00 \%$ for starch, $46.42 \%$ samples were detected for water, $32.14 \%$ for sugar, $10.71 \%$ for glucose $17.85 \%$ samples detected for salt and $21.42 \%$ were detected positive for neutralizers.

Adulteration of water, sugar and salt found in higher percentage than other adulteration in organized dairy farm milk samples. The reasons for this use of adulterants that, it may be easily available in market and cheaper than other adulterants. Sugar and salt added in milk to mask the effect of added water (Sharma et al., 2015). 
Table.1 Resources of milk samples

\begin{tabular}{|l|l|l|}
\hline $\begin{array}{l}\text { Name of } \\
\text { Brands Milk }\end{array}$ & $\begin{array}{l}\text { Name of Organized } \\
\text { Dairy Farm }\end{array}$ & $\begin{array}{l}\text { Name of Traditional } \\
\text { Vendors Milk }\end{array}$ \\
\hline Amul & B.H.U. dairy farm & Vendor 1 \\
\hline Gyan & Surbhi dairy farm & Vendor 2 \\
\hline Kashi & Siyaram dairy farm & Vendor 3 \\
\hline Parag & Nature dairy farm & Vendor 4 \\
\hline Mother dairy & Alok dairy farm & Vendor 5 \\
\hline Paras & Maa Amby dairy farm & Vendor 6 \\
\hline Suddh & Dudh ganga dairy farm & Vendor 7 \\
\hline
\end{tabular}

Table.2 Total number of samples

\begin{tabular}{|l|c|c|c|}
\hline Name of resources & Samples & Replication & Total samples \\
\hline Branded full cream milk samples & 7 & 4 & 28 \\
\hline Branded toned milk & 7 & 4 & 28 \\
\hline Organized dairy farm cow milk & 7 & 4 & 28 \\
\hline Organized dairy farm buffalo milk & 7 & 4 & 28 \\
\hline Traditional vendors cow milk & 7 & 4 & 28 \\
\hline Traditional vendors buffalo milk & 7 & 4 & 28 \\
\hline
\end{tabular}

Adulteration in organized dairy farm buffalo milk samples

All 28 samples from organized dairy farm were found to be negative for urea, ammonium fertilizer, nitrate fertilizers, starch $\&$ neutralizer and hydrogen peroxide, while 3 milk samples were positive for urea, 8 milk samples positive for starch and 15 samples were positive for water, 12 milk samples were positive for sugar, 4 milk samples were positive for glucose, 9 samples was found positive for salt and 7 milk samples were positive for neutralizers.

Out of total analysis organized dairy farm buffalo milk samples, $10.71 \%$ samples were detected positive for urea, $28.57 \%$ for starch, $53.57 \%$ samples were detected for water, $42.85 \%$ for sugar, $14.28 \%$ for glucose $32.14 \%$ samples detected for salt and $25.00 \%$ were detected positive for neutralizers. Adulteration of water, sugar and salt found in higher percentage than other adulteration in organized dairy farm milk samples. The reasons for this use of adulterants that, it may be easily available in market and cheaper than other adulterants. Sugar and salt added in milk to mask the effect of added water (Sharma et al., 2015).

\section{Adulteration in traditional vendors cow milk sample}

All 28 samples from traditional vendors milk samples were found to be negative for urea, ammonium fertilizer, nitrate fertilizers neutralizer and hydrogen peroxide, while 12 milk samples positive for starch and 21 samples were positive for water, 19 samples were positive for sugar, 7 milk samples were positive for glucose, and 14 samples was found positive for salt. 
Table.3 Detection of adulteration in branded full cream milk samples

\begin{tabular}{|c|c|c|c|c|c|c|c|c|c|c|}
\hline Adulterants & & ilizers & & Starch & Water & Sugar & Glucose & Salt & Neutral & $\mathrm{H}_{2} \mathrm{O}_{2}$ \\
\hline Samples & Urea & $\mathbf{N H}_{3}$ & $\mathbf{N O}_{3}$ & & & & & & & \\
\hline $\mathbf{A}_{1}$ & -ve & -ve & -ve & -ve & -ve & -ve & -ve & -ve & -ve & -ve \\
\hline $\mathbf{A}_{1}$ & -ve & -ve & -ve & -ve & -ve & -ve & -ve & -ve & -ve & -ve \\
\hline $\mathbf{A}_{1}$ & -ve & -ve & -ve & -ve & -ve & -ve & -ve & -ve & -ve & -ve \\
\hline $\mathbf{A}_{1}$ & -ve & -ve & -ve & -ve & -ve & -ve & -ve & -ve & -ve & -ve \\
\hline $\mathbf{G}_{2}$ & -ve & -ve & -ve & -ve & -ve & -ve & -ve & -ve & -ve & -ve \\
\hline $\mathbf{G}_{2}$ & -ve & -ve & -ve & -ve & -ve & -ve & -ve & -ve & -ve & -ve \\
\hline $\mathbf{G}_{2}$ & -ve & -ve & -ve & -ve & -ve & -ve & -ve & -ve & -ve & -ve \\
\hline $\mathbf{G}_{2}$ & -ve & -ve & -ve & -ve & -ve & -ve & -ve & -ve & -ve & -ve \\
\hline $\mathbf{K}_{\mathbf{3}}$ & -ve & -ve & -ve & -ve & -ve & -ve & -ve & -ve & -ve & -ve \\
\hline $\mathbf{K}_{\mathbf{3}}$ & -ve & -ve & -ve & -ve & -ve & -ve & -ve & -ve & -ve & -ve \\
\hline $\mathbf{K}_{3}$ & -ve & -ve & -ve & -ve & -ve & -ve & -ve & -ve & -ve & -ve \\
\hline $\mathbf{K}_{3}$ & -ve & -ve & -ve & -ve & -ve & -ve & -ve & -ve & -ve & -ve \\
\hline $\mathbf{P G}_{4}$ & -ve & -ve & -ve & -ve & -ve & -ve & -ve & -ve & -ve & -ve \\
\hline $\mathbf{P G}_{4}$ & -ve & -ve & -ve & -ve & -ve & -ve & -ve & -ve & -ve & -ve \\
\hline $\mathbf{P G}_{4}$ & -ve & -ve & -ve & $-v e$ & $-v e$ & -ve & -ve & -ve & -ve & -ve \\
\hline $\mathbf{P G}_{4}$ & -ve & -ve & -ve & -ve & -ve & -ve & -ve & -ve & -ve & -ve \\
\hline $\mathrm{MD}_{5}$ & -ve & -ve & -ve & -ve & -ve & -ve & -ve & -ve & -ve & -ve \\
\hline $\mathbf{M D}_{5}$ & -ve & -ve & -ve & -ve & -ve & -ve & -ve & -ve & -ve & -ve \\
\hline $\mathrm{MD}_{5}$ & -ve & -ve & -ve & -ve & -ve & -ve & -ve & -ve & -ve & -ve \\
\hline $\mathbf{M D}_{5}$ & -ve & -ve & -ve & -ve & -ve & -ve & -ve & -ve & -ve & -ve \\
\hline $\mathbf{P S}_{6}$ & -ve & -ve & -ve & -ve & -ve & -ve & -ve & -ve & -ve & -ve \\
\hline $\mathbf{P S}_{6}$ & -ve & -ve & -ve & -ve & -ve & -ve & -ve & -ve & -ve & -ve \\
\hline $\mathbf{P S}_{6}$ & -ve & -ve & -ve & -ve & -ve & -ve & -ve & -ve & -ve & -ve \\
\hline $\mathbf{P S}_{6}$ & -ve & -ve & -ve & -ve & -ve & -ve & -ve & -ve & -ve & -ve \\
\hline $\mathrm{SD}_{7}$ & -ve & -ve & -ve & -ve & -ve & -ve & -ve & -ve & -ve & -ve \\
\hline $\mathrm{SD}_{7}$ & -ve & -ve & -ve & -ve & -ve & -ve & -ve & -ve & -ve & -ve \\
\hline $\mathrm{SD}_{7}$ & -ve & -ve & -ve & -ve & -ve & -ve & -ve & -ve & -ve & -ve \\
\hline $\mathrm{SD}_{7}$ & -ve & -ve & -ve & -ve & -ve & -ve & -ve & -ve & -ve & -ve \\
\hline Samples +ve & 00 & 00 & 00 & 00 & 00 & 00 & 00 & 00 & 00 & 00 \\
\hline Percentage & 00 & 00 & 00 & 00 & 00 & 00 & 00 & 00 & 00 & 00 \\
\hline
\end{tabular}


Table.4 Detection of adulteration in branded toned milk samples

\begin{tabular}{|c|c|c|c|c|c|c|c|c|c|c|}
\hline \multirow{2}{*}{\begin{tabular}{|l|} 
Adulterants \\
Samples
\end{tabular}} & \multicolumn{3}{|c|}{ Fertilizers } & \multirow[t]{2}{*}{ Starch } & \multirow[t]{2}{*}{ Water } & \multirow[t]{2}{*}{ Sugar } & \multirow{2}{*}{$\begin{array}{l}\text { Gluco } \\
\text { se }\end{array}$} & \multirow[t]{2}{*}{ Salt } & \multirow{2}{*}{$\begin{array}{l}\text { Neutral } \\
\text { izers }\end{array}$} & \multirow[t]{2}{*}{$\mathbf{H}_{2} \mathbf{O}_{2}$} \\
\hline & Urea & $\mathbf{N H}_{3}$ & $\mathrm{NO}_{3}$ & & & & & & & \\
\hline $\mathbf{A}_{8}$ & $-v e$ & -ve & -ve & -ve & -ve & -ve & -ve & -ve & -ve & -ve \\
\hline $\mathbf{A}_{8}$ & -ve & -ve & -ve & -ve & -ve & -ve & -ve & -ve & -ve & -ve \\
\hline $\mathbf{A}_{8}$ & $-v e$ & $-\mathrm{ve}$ & -ve & $-v e$ & -ve & -ve & -ve & $-\mathrm{ve}$ & -ve & $-\mathrm{ve}$ \\
\hline $\mathbf{A}_{8}$ & -ve & -ve & -ve & -ve & -ve & -ve & -ve & -ve & -ve & -ve \\
\hline G9 & -ve & -ve & -ve & -ve & -ve & -ve & -ve & -ve & -ve & -ve \\
\hline $\mathbf{G}_{9}$ & $-\mathrm{ve}$ & -ve & -ve & -ve & -ve & -ve & -ve & $-\mathrm{ve}$ & -ve & $-\mathrm{ve}$ \\
\hline $\mathbf{G}_{9}$ & -ve & -ve & -ve & -ve & -ve & -ve & -ve & -ve & -ve & -ve \\
\hline $\mathbf{G}_{9}$ & -ve & -ve & -ve & -ve & -ve & -ve & -ve & $-\mathrm{ve}$ & -ve & $-\mathrm{ve}$ \\
\hline$K_{10}$ & -ve & -ve & -ve & -ve & -ve & -ve & -ve & -ve & -ve & -ve \\
\hline $\mathbf{K}_{10}$ & -ve & -ve & -ve & -ve & -ve & -ve & -ve & -ve & -ve & -ve \\
\hline $\mathbf{K}_{10}$ & -ve & -ve & -ve & -ve & -ve & -ve & -ve & -ve & -ve & -ve \\
\hline $\mathbf{K}_{10}$ & -ve & -ve & -ve & -ve & -ve & -ve & -ve & -ve & -ve & -ve \\
\hline $\mathbf{P G}_{11}$ & -ve & -ve & -ve & -ve & -ve & -ve & -ve & -ve & -ve & -ve \\
\hline $\mathbf{P G}_{11}$ & -ve & -ve & -ve & -ve & -ve & -ve & -ve & -ve & -ve & -ve \\
\hline $\mathbf{P G}_{11}$ & -ve & -ve & -ve & -ve & -ve & -ve & -ve & -ve & -ve & -ve \\
\hline $\mathbf{P G}_{11}$ & -ve & -ve & -ve & -ve & -ve & -ve & -ve & -ve & -ve & -ve \\
\hline $\mathrm{MD}_{12}$ & -ve & -ve & -ve & -ve & -ve & -ve & -ve & -ve & -ve & -ve \\
\hline $\mathrm{MD}_{12}$ & -ve & -ve & -ve & -ve & -ve & -ve & -ve & -ve & -ve & -ve \\
\hline $\mathrm{MD}_{12}$ & -ve & -ve & -ve & -ve & -ve & -ve & -ve & -ve & -ve & -ve \\
\hline $\mathbf{M D}_{12}$ & -ve & -ve & -ve & -ve & -ve & -ve & -ve & -ve & -ve & -ve \\
\hline $\mathbf{P S}_{13}$ & -ve & -ve & -ve & -ve & -ve & -ve & -ve & -ve & -ve & -ve \\
\hline $\mathbf{P S}_{13}$ & -ve & -ve & -ve & -ve & -ve & -ve & -ve & -ve & -ve & -ve \\
\hline $\mathbf{P S}_{13}$ & -ve & -ve & -ve & -ve & -ve & -ve & -ve & -ve & -ve & -ve \\
\hline $\mathbf{P S}_{13}$ & -ve & -ve & -ve & -ve & -ve & -ve & -ve & -ve & -ve & -ve \\
\hline $\mathrm{SD}_{14}$ & -ve & -ve & -ve & -ve & -ve & -ve & -ve & -ve & -ve & -ve \\
\hline $\mathrm{SD}_{14}$ & -ve & -ve & -ve & -ve & -ve & -ve & -ve & -ve & -ve & -ve \\
\hline $\mathrm{SD}_{14}$ & -ve & -ve & -ve & -ve & -ve & -ve & -ve & -ve & $\mathrm{Ve}$ & -ve \\
\hline $\mathrm{SD}_{14}$ & -ve & -ve & -ve & -ve & -ve & -ve & -ve & -ve & -ve & -ve \\
\hline Samples +ve & 00 & 00 & 00 & 00 & 00 & 00 & 00 & 00 & 00 & 00 \\
\hline Percentage & 00 & 00 & 00 & 00 & 00 & 00 & 00 & 00 & 00 & 00 \\
\hline
\end{tabular}


Table.5 Detection of adulteration in organized dairy farm cow milk samples

\begin{tabular}{|c|c|c|c|c|c|c|c|c|c|c|}
\hline Adulterants & \multicolumn{3}{|c|}{ fertilizers } & \multirow[t]{2}{*}{ Starch } & \multirow[t]{2}{*}{ Water } & \multirow[t]{2}{*}{ Sugar } & \multirow[t]{2}{*}{ Glucose } & \multirow[t]{2}{*}{ Salt } & \multirow{2}{*}{$\begin{array}{l}\text { Neutr } \\
\text { alizers }\end{array}$} & \multirow[t]{2}{*}{$\mathrm{H}_{2} \mathrm{O}_{2}$} \\
\hline Samples & Urea & $\mathbf{N H}_{3}$ & $\mathrm{NO}_{3}$ & & & & & & & \\
\hline $\mathbf{B}_{1}$ & -ve & -ve & -ve & -ve & -ve & -ve & -ve & -ve & -ve & -ve \\
\hline $\mathbf{B}_{1}$ & -ve & -ve & -ve & -ve & $-\mathrm{ve}$ & -ve & -ve & $-\mathrm{ve}$ & -ve & -ve \\
\hline $\mathbf{B}_{1}$ & -ve & -ve & -ve & -ve & -ve & -ve & -ve & -ve & -ve & -ve \\
\hline $\mathbf{B}_{1}$ & -ve & -ve & -ve & -ve & -ve & -ve & -ve & -ve & -ve & -ve \\
\hline $\mathbf{S B}_{2}$ & -ve & $-v e$ & -ve & $-v e$ & -ve & $-v e$ & -ve & -ve & -ve & -ve \\
\hline $\mathbf{S B}_{2}$ & -ve & -ve & -ve & -ve & -ve & -ve & -ve & -ve & -ve & - ve \\
\hline $\mathbf{S B}_{2}$ & -ve & -ve & -ve & $-\mathrm{ve}$ & $-\mathrm{ve}$ & -ve & -ve & $-\mathrm{ve}$ & -ve & -ve \\
\hline $\mathbf{S B}_{2}$ & -ve & -ve & -ve & -ve & -ve & -ve & -ve & -ve & $+v e$ & - ve \\
\hline $\mathbf{S Y}_{3}$ & -ve & -ve & $-\mathrm{ve}$ & -ve & $+v e$ & $+v e$ & -ve & -ve & -ve & -ve \\
\hline $\mathrm{SY}_{3}$ & -ve & -ve & -ve & $+v e$ & $+v e$ & -ve & -ve & -ve & -ve & -ve \\
\hline $\mathbf{S Y}_{3}$ & -ve & -ve & -ve & $+v e$ & -ve & -ve & -ve & -ve & $+v e$ & - ve \\
\hline $\mathrm{SY}_{3}$ & -ve & -ve & -ve & -ve & +ve & +ve & -ve & +ve & -ve & - ve \\
\hline $\mathbf{N}_{4}$ & -ve & -ve & -ve & -ve & -ve & -ve & -ve & $+v e$ & -ve & - ve \\
\hline $\mathbf{N}_{4}$ & -ve & - ve & -ve & $+\mathrm{ve}$ & $-v e$ & $-v e$ & -ve & -ve & +ve & - ve \\
\hline $\mathbf{N}_{4}$ & -ve & -ve & -ve & -ve & $+v e$ & $+v e$ & -ve & -ve & -ve & -ve \\
\hline $\mathbf{N}_{4}$ & -ve & - ve & -ve & $+\mathrm{ve}$ & $+v e$ & $-v e$ & -ve & -ve & -ve & - ve \\
\hline $\mathbf{A K} \mathbf{K}_{5}$ & $+v e$ & -ve & -ve & -ve & -ve & -ve & $+v e$ & -ve & -ve & $-\mathrm{ve}$ \\
\hline $\mathbf{A K}_{5}$ & -ve & -ve & -ve & -ve & $+v e$ & $+v e$ & -ve & -ve & -ve & -ve \\
\hline $\mathbf{A K}_{5}$ & $+v e$ & -ve & -ve & -ve & -ve & -ve & -ve & $+v e$ & -ve & - ve \\
\hline $\mathbf{A K} \mathbf{K}_{5}$ & -ve & -ve & -ve & -ve & +ve & +ve & -ve & $-v e$ & -ve & -ve \\
\hline $\mathbf{M A}_{6}$ & -ve & $-v e$ & -ve & $+v e$ & -ve & -ve & -ve & -ve & -ve & -ve \\
\hline $\mathbf{M A}_{6}$ & -ve & -ve & -ve & -ve & $+\mathrm{ve}$ & +ve & -ve & -ve & $+v e$ & - ve \\
\hline $\mathbf{M A}_{6}$ & -ve & -ve & -ve & $+v e$ & $+v e$ & -ve & -ve & -ve & $+v e$ & -ve \\
\hline $\mathbf{M A}_{6}$ & -ve & - ve & -ve & -ve & $+v e$ & $+v e$ & +ve & -ve & -ve & -ve \\
\hline $\mathbf{D G}_{7}$ & $+v e$ & -ve & -ve & $+v e$ & $+v e$ & $+v e$ & -ve & -ve & -ve & - ve \\
\hline $\mathbf{D G}_{7}$ & $+v e$ & -ve & -ve & -ve & $+v e$ & -ve & +ve & $+v e$ & $+v e$ & -ve \\
\hline $\mathbf{D G}_{7}$ & -ve & -ve & -ve & -ve & $+v e$ & $+v e$ & -ve & -ve & -ve & -ve \\
\hline $\mathbf{D G}_{7}$ & -ve & -ve & -ve & -ve & -ve & -ve & -ve & $+\mathrm{ve}$ & -ve & -ve \\
\hline Sample +ve & 04 & 00 & 00 & 07 & 13 & 09 & 03 & 05 & 06 & 00 \\
\hline Percentage & 14.28 & 00 & 00 & 25.00 & 46.42 & 32.14 & 10.71 & 17.85 & 21.42 & 00 \\
\hline
\end{tabular}


Table.6 Detection of adulteration in organized dairy farm buffalo milk samples

\begin{tabular}{|c|c|c|c|c|c|c|c|c|c|c|}
\hline Adulterants & & ertiliz & & Starch & Water & Sugar & Glucose & Salt & Neutr & $\mathrm{H}_{2} \mathrm{O}_{2}$ \\
\hline Samples & Urea & $\mathbf{N H}_{3}$ & $\mathbf{N O}_{3}$ & & & & & & alizers & \\
\hline $\mathbf{B}_{8}$ & $-\mathrm{ve}$ & -ve & -ve & -ve & -ve & -ve & -ve & -ve & -ve & -ve \\
\hline $\mathbf{B}_{8}$ & -ve & -ve & -ve & -ve & $-v e$ & $-v e$ & -ve & -ve & -ve & -ve \\
\hline $\mathbf{B}_{8}$ & -ve & -ve & -ve & -ve & -ve & -ve & -ve & -ve & -ve & -ve \\
\hline $\mathbf{B}_{8}$ & -ve & -ve & -ve & -ve & -ve & -ve & -ve & -ve & -ve & -ve \\
\hline SB9 & -ve & -ve & -ve & $+v e$ & $-v e$ & +ve & -ve & $+v e$ & -ve & -ve \\
\hline SB9 $_{9}$ & -ve & -ve & -ve & $+v e$ & -ve & -ve & $+v e$ & - ve & -ve & -ve \\
\hline SB9 $_{9}$ & -ve & -ve & -ve & -ve & $+v e$ & -ve & -ve & -ve & $+v e$ & -ve \\
\hline SB9 $_{9}$ & $+v e$ & -ve & -ve & -ve & $+v e$ & $+v e$ & -ve & -ve & -ve & -ve \\
\hline $\mathrm{SY}_{10}$ & -ve & -ve & -ve & -ve & -ve & -ve & $+v e$ & $+v e$ & -ve & -ve \\
\hline $\mathrm{SY}_{10}$ & -ve & -ve & -ve & -ve & $+\mathrm{ve}$ & -ve & -ve & - ve & $+v e$ & -ve \\
\hline $\mathrm{SY}_{10}$ & -ve & -ve & -ve & $+v e$ & -ve & +ve & -ve & -ve & -ve & -ve \\
\hline $\mathrm{SY}_{10}$ & +ve & -ve & -ve & +ve & $+\mathrm{ve}$ & +ve & -ve & - ve & -ve & -ve \\
\hline $\mathbf{N}_{11}$ & $+v e$ & -ve & -ve & -ve & -ve & -ve & -ve & $+v e$ & -ve & -ve \\
\hline $\mathbf{N}_{11}$ & -ve & -ve & -ve & -ve & $+v e$ & +ve & -ve & -ve & -ve & -ve \\
\hline $\mathbf{N}_{11}$ & -ve & -ve & -ve & -ve & -ve & -ve & +ve & $+\mathrm{ve}$ & +ve & -ve \\
\hline $\mathbf{N}_{11}$ & -ve & -ve & -ve & -ve & $+\mathrm{ve}$ & $+\mathrm{ve}$ & -ve & -ve & -ve & -ve \\
\hline $\mathbf{A} \mathbf{K}_{12}$ & -ve & -ve & -ve & -ve & -ve & -ve & -ve & $+v e$ & -ve & -ve \\
\hline $\mathbf{A} \mathbf{K}_{12}$ & -ve & -ve & -ve & $+v e$ & $+\mathrm{ve}$ & +ve & -ve & -ve & -ve & -ve \\
\hline $\mathbf{A} K_{12}$ & -ve & -ve & -ve & -ve & $+\mathrm{ve}$ & -ve & -ve & $+\mathrm{ve}$ & -ve & -ve \\
\hline $\mathbf{A} \mathbf{K}_{13}$ & -ve & -ve & -ve & -ve & $+\mathrm{ve}$ & -ve & -ve & -ve & -ve & -ve \\
\hline $\mathbf{M A}_{13}$ & -ve & -ve & -ve & +ve & -ve & $+v e$ & -ve & - ve & +ve & -ve \\
\hline $\mathbf{M A}_{13}$ & -ve & -ve & -ve & -ve & $+\mathrm{ve}$ & $+v e$ & -ve & -ve & -ve & -ve \\
\hline $\mathbf{M A}_{13}$ & -ve & -ve & -ve & -ve & -ve & -ve & -ve & $+v e$ & -ve & -ve \\
\hline $\mathbf{M A}_{13}$ & -ve & -ve & -ve & $+v e$ & $+\mathrm{ve}$ & +ve & -ve & -ve & +ve & -ve \\
\hline $\mathrm{DG}_{14}$ & -ve & -ve & -ve & -ve & $+\mathrm{ve}$ & -ve & $+\mathrm{ve}$ & -ve & $+v e$ & -ve \\
\hline $\mathrm{DG}_{14}$ & $+v e$ & -ve & -ve & $+v e$ & $+v e$ & $+v e$ & -ve & $+v e$ & -ve & -ve \\
\hline $\mathrm{DG}_{14}$ & -ve & -ve & -ve & -ve & $+v e$ & +ve & -ve & -ve & $+\mathrm{ve}$ & -ve \\
\hline $\mathrm{DG}_{14}$ & -ve & -ve & -ve & -ve & +ve & -ve & -ve & +ve & -ve & -ve \\
\hline Samples +ve & 03 & 00 & 00 & 08 & 15 & 12 & 04 & 09 & 07 & 00 \\
\hline Percentage & 10.71 & 00 & 00 & 28.57 & 53.57 & 42.85 & 14.28 & 32.14 & 25.00 & 00 \\
\hline
\end{tabular}


Table.7 Detection of adulteration in traditional vendors cow milk sample

\begin{tabular}{|c|c|c|c|c|c|c|c|c|c|c|}
\hline Adulterants & \multicolumn{3}{|c|}{ Fertilizers } & \multirow[t]{2}{*}{ Starch } & \multirow[t]{2}{*}{ Water } & \multirow[t]{2}{*}{ Sugar } & \multirow[t]{2}{*}{ Glucose } & \multirow[t]{2}{*}{ Salt } & \multirow{2}{*}{$\begin{array}{l}\text { Neutr } \\
\text { alizers }\end{array}$} & \multirow[t]{2}{*}{$\mathrm{H}_{2} \mathrm{O}_{2}$} \\
\hline Samples & Urea & $\mathbf{N H}_{3}$ & $\mathbf{N O}_{3}$ & & & & & & & \\
\hline$V_{1}$ & -ve & -ve & -ve & + ve & +ve & $+\mathrm{ve}$ & -ve & $+\mathrm{ve}$ & -ve & -ve \\
\hline$V_{1}$ & -ve & -ve & -ve & -ve & $+v e$ & -ve & $+v e$ & -ve & -ve & -ve \\
\hline$V_{1}$ & -ve & -ve & -ve & $+v e$ & + ve & + ve & -ve & + ve & -ve & -ve \\
\hline $\mathbf{V}_{1}$ & -ve & -ve & -ve & -ve & $+v e$ & $+v e$ & $+\mathrm{ve}$ & -ve & -ve & -ve \\
\hline $\mathbf{V}_{2}$ & -ve & -ve & -ve & $+v e$ & -ve & $+v e$ & -ve & $+v e$ & -ve & -ve \\
\hline$V_{2}$ & -ve & -ve & - ve & -ve & +ve & $+v e$ & -ve & $+\mathrm{ve}$ & -ve & -ve \\
\hline $\mathbf{V}_{2}$ & -ve & -ve & -ve & -ve & $+v e$ & $+v e$ & -ve & -ve & -ve & -ve \\
\hline $\mathbf{V}_{2}$ & -ve & -ve & -ve & $+v e$ & +ve & -ve & +ve & -ve & -ve & -ve \\
\hline $\mathbf{V}_{3}$ & -ve & -ve & -ve & -ve & $+v e$ & $+v e$ & -ve & $+v e$ & -ve & -ve \\
\hline $\mathbf{V}_{3}$ & -ve & -ve & -ve & -ve & $+\mathrm{ve}$ & $+\mathrm{ve}$ & -ve & -ve & -ve & -ve \\
\hline $\mathbf{V}_{3}$ & -ve & -ve & -ve & $+v e$ & $+v e$ & -ve & -ve & $+v e$ & -ve & -ve \\
\hline $\mathbf{V}_{3}$ & -ve & -ve & -ve & -ve & -ve & $+v e$ & -ve & -ve & -ve & -ve \\
\hline $\mathbf{V}_{4}$ & -ve & -ve & -ve & $+v e$ & +ve & $+v e$ & -ve & $+v e$ & -ve & -ve \\
\hline $\mathbf{V}_{4}$ & -ve & -ve & -ve & $+v e$ & -ve & -ve & $+\mathrm{ve}$ & -ve & -ve & -ve \\
\hline $\mathbf{V}_{4}$ & -ve & -ve & -ve & -ve & +ve & $+\mathrm{ve}$ & -ve & $+\mathrm{ve}$ & -ve & -ve \\
\hline $\mathbf{V}_{4}$ & -ve & -ve & -ve & $+v e$ & -ve & -ve & -ve & -ve & -ve & -ve \\
\hline$V_{5}$ & -ve & -ve & -ve & -ve & $+\mathrm{ve}$ & $+\mathrm{ve}$ & -ve & $+\mathrm{ve}$ & -ve & -ve \\
\hline$V_{5}$ & -ve & -ve & -ve & $+\mathrm{ve}$ & -ve & -ve & $+\mathrm{ve}$ & $+\mathrm{ve}$ & -ve & -ve \\
\hline$V_{5}$ & -ve & -ve & -ve & -ve & +ve & $+\mathrm{ve}$ & -ve & -ve & -ve & -ve \\
\hline$V_{5}$ & -ve & -ve & -ve & -ve & +ve & +ve & -ve & +ve & -ve & -ve \\
\hline$V_{6}$ & -ve & -ve & -ve & -ve & $+\mathrm{ve}$ & -ve & -ve & -ve & -ve & -ve \\
\hline$V_{6}$ & -ve & -ve & -ve & -ve & $+v e$ & $+\mathrm{ve}$ & -ve & $+v e$ & -ve & -ve \\
\hline$V_{6}$ & -ve & -ve & -ve & +ve & -ve & -ve & -ve & -ve & -ve & -ve \\
\hline$V_{6}$ & -ve & -ve & -ve & -ve & $+\mathrm{ve}$ & $+\mathrm{ve}$ & -ve & $+\mathrm{ve}$ & -ve & -ve \\
\hline $\mathrm{V}_{7}$ & -ve & -ve & -ve & $+v e$ & +ve & $+\mathrm{ve}$ & -ve & -ve & -ve & -ve \\
\hline $\mathbf{V}_{7}$ & -ve & -ve & -ve & -ve & $+v e$ & -ve & $+\mathrm{ve}$ & -ve & -ve & -ve \\
\hline$V_{7}$ & -ve & -ve & -ve & +ve & +ve & +ve & -ve & $+\mathrm{ve}$ & -ve & -ve \\
\hline$V_{7}$ & -ve & -ve & -ve & -ve & -ve & $+\mathrm{ve}$ & $+\mathrm{ve}$ & -ve & -ve & -ve \\
\hline Samples +ve & 00 & 00 & 00 & 12 & 21 & 19 & 07 & 14 & 00 & 00 \\
\hline Percentage & 00 & 00 & 00 & 42.85 & 75.00 & 67.85 & 25.00 & 50.00 & 00 & 00 \\
\hline
\end{tabular}


Table.8 Detection of adulteration in traditional vendors buffalo milk samples

\begin{tabular}{|c|c|c|c|c|c|c|c|c|c|c|}
\hline Adulterants & Urea & fertili & zers & Starch & Water & Sugar & Glucose & Salt & $\begin{array}{l}\text { Neutr } \\
\text { alizers }\end{array}$ & $\mathrm{H}_{2} \mathrm{O}_{2}$ \\
\hline Samples & & $\mathbf{N H}_{3}$ & $\mathrm{NO}_{3}$ & & & & & & & \\
\hline V8 & -ve & -ve & $-v e$ & $+v e$ & $+v e$ & $+v e$ & -ve & $+v e$ & -ve & -ve \\
\hline V8 & -ve & -ve & -ve & -ve & $+v e$ & -ve & +ve & -ve & -ve & -ve \\
\hline V8 & -ve & -ve & -ve & -ve & $+v e$ & +ve & -ve & +ve & -ve & -ve \\
\hline V8 & -ve & -ve & -ve & -ve & $+v e$ & $+v e$ & -ve & -ve & -ve & -ve \\
\hline V9 & -ve & -ve & -ve & +ve & $+v e$ & +ve & -ve & +ve & -ve & -ve \\
\hline V9 & -ve & -ve & -ve & -ve & $+v e$ & $+v e$ & -ve & + ve & -ve & -ve \\
\hline V9 & -ve & -ve & -ve & -ve & $+v e$ & +ve & $-v e$ & -ve & -ve & -ve \\
\hline V9 & -ve & -ve & -ve & $+v e$ & $+v e$ & -ve & +ve & $+\mathrm{ve}$ & -ve & -ve \\
\hline V10 & -ve & -ve & -ve & -ve & $+v e$ & $+v e$ & -ve & $+v e$ & -ve & -ve \\
\hline V10 & -ve & -ve & -ve & -ve & $+v e$ & $+v e$ & $-v e$ & -ve & -ve & -ve \\
\hline V10 & -ve & -ve & -ve & $+v e$ & $+v e$ & -ve & $-v e$ & $+v e$ & -ve & -ve \\
\hline V10 & -ve & -ve & -ve & -ve & $+v e$ & $+v e$ & -ve & - ve & -ve & -ve \\
\hline V11 & -ve & -ve & -ve & +ve & $+v e$ & +ve & -ve & +ve & -ve & -ve \\
\hline V11 & -ve & -ve & -ve & -ve & -ve & $+v e$ & $+v e$ & -ve & -ve & -ve \\
\hline V11 & -ve & -ve & -ve & -ve & $+v e$ & +ve & -ve & +ve & -ve & -ve \\
\hline V11 & -ve & -ve & -ve & +ve & $+v e$ & $+v e$ & -ve & -ve & -ve & -ve \\
\hline V12 & -ve & -ve & -ve & -ve & $+v e$ & +ve & -ve & +ve & -ve & -ve \\
\hline V12 & -ve & -ve & -ve & +ve & -ve & -ve & $+v e$ & +ve & -ve & -ve \\
\hline V12 & -ve & -ve & -ve & -ve & $+v e$ & $+\mathrm{ve}$ & -ve & -ve & -ve & -ve \\
\hline V12 & -ve & -ve & -ve & -ve & $+v e$ & $+v e$ & -ve & + ve & -ve & -ve \\
\hline V13 & -ve & -ve & -ve & -ve & $+\mathrm{ve}$ & -ve & -ve & -ve & -ve & -ve \\
\hline V13 & -ve & -ve & -ve & -ve & $+\mathrm{ve}$ & +ve & -ve & +ve & -ve & -ve \\
\hline V13 & -ve & -ve & -ve & $+v e$ & -ve & -ve & -ve & -ve & -ve & -ve \\
\hline V13 & -ve & -ve & -ve & -ve & $+v e$ & $+v e$ & -ve & +ve & -ve & -ve \\
\hline V14 & -ve & -ve & -ve & +ve & $+v e$ & +ve & -ve & +ve & -ve & -ve \\
\hline V14 & -ve & -ve & -ve & -ve & $+v e$ & -ve & -ve & +ve & -ve & -ve \\
\hline V14 & -ve & -ve & -ve & +ve & $+v e$ & +ve & -ve & +ve & -ve & -ve \\
\hline V14 & -ve & -ve & -ve & -ve & $+v e$ & $+\mathrm{ve}$ & $+v e$ & -ve & -ve & -ve \\
\hline Samples +ve & 00 & 00 & 00 & 10 & 25 & 21 & 05 & 17 & 00 & 00 \\
\hline Percentage & 00 & 00 & 00 & 35.71 & 89.28 & 75.00 & 17.85 & 60.71 & 00 & 00 \\
\hline
\end{tabular}

Out of total analysis traditional vendors cow milk samples, $42.85 \%$ for starch, $75.00 \%$ samples were detected for water, $67.85 \%$ for sugar, $25.00 \%$ for glucose and $50.00 \%$ samples detected for salt. Adulteration of water, sugar and salt found in higher percentage than other adulteration in traditional vendors milk samples. The reasons for this use of adulterants that, it may be easily available added water (Sharma et al., 2015). On the basis of the present investigation, it may be concluded that Out of 
168 milk samples, 44.04\% milk samples were found to be with water, $4.16 \%$ with urea, $22.02 \%$ with starch, $36.30 \%$ with sugar, $11.30 \%$ with glucose, $26.78 \%$ with salt and $7.38 \%$ with neutralizers. Total 28 milk samples from branded were found to be negative for urea, ammonium fertilizer, nitrate fertilizers, starch, salt, neutralizer and hydrogen peroxide. Total 28 milk samples from analysis organized dairy farm cow milk samples, $14.28 \%$ samples were detected positive for urea, $25.00 \%$ for starch, $46.42 \%$ samples were detected for water, $32.14 \%$ for sugar, $10.71 \%$ for glucose $17.85 \%$ samples detected for salt and $21.42 \%$ were detected positive for neutralizers.

Out of 28 milk samples from analysis organized dairy farm buffalo milk samples, $10.71 \%$ samples were detected positive for urea, $28.57 \%$ for starch, $53.57 \%$ samples were detected for water, $42.85 \%$ for sugar, $14.28 \%$ for glucose $32.14 \%$ samples detected for salt and $25.00 \%$ were detected positive for neutralizers. Out of 28 milk samples from analysis traditional vendors cow milk samples, $42.85 \%$ for starch, $75.00 \%$ samples were detected for water, $67.85 \%$ for sugar, $25.00 \%$ for glucose and $50.00 \%$ samples detected for salt.

Out of 28 milk samples from analysis traditional vendors buffalo milk samples, $35.71 \%$ samples were detected positive for starch, $89.28 \%$ for water, $75.00 \%$ for sugar,
$17.85 \%$ for glucose and $60.71 \%$ samples detected for salt. Adulteration of water, sugar and salt found in higher percentage than other adulteration in traditional vendors milk samples. These results are only indicative and require further experimentation to arrive at some more consistent conclusion.

\section{References}

Awan A., Naseer M., Iqbal A., Ali M., Iqbal R. and Iqbal F. (2014). A study on chemical composition and detection of chemical adulteration in tetra pack milk samples commercially available in Multan. Pakistan journal of pharmaceutical sciences, 27 (1): $\quad$ 183-186.

Ellis F. and Sumberg J. (1998). Food production, urban areas and policy responses. World Development, 26 (2): 213-225.

FSSAI (2011). Food safety and standards (food products standards and food additives) regulations. www. fssai. gov. in assessed on 29. 03. 2019.

NDDB (2017). National dairy development board. www. nddb. coop.

Sharma, R., Mann, B., Satya, K., and Nanda D.K. (2015). Rapid diagnostic test for the detection of milk adulterations, current status, CAFT, DT, Nov. 28 Dec. 18, 2015. ICAR NDRI publication. 131-135.

\section{How to cite this article:}

Dwarki Lal, D. C. Rai, Vinod Bhateshwar, Hitesh Muwal and Govind. 2020. Detection of Adulteration and Quality Evaluation of Market Milk and Raw Milk Collected from Market in Varanasi City. Int.J.Curr.Microbiol.App.Sci. 9(03): 2984-2996. doi: https://doi.org/10.20546/ijcmas.2020.903.342 